\title{
Chang E III Soft Landing Optimal Control Strategy Design
}

\author{
Xinjie $\operatorname{Han}^{\mathrm{a}}$ \\ North China Electric Power University, Baoding 071000, China \\ a2399037135@qq.com
}

Keywords: Two-point boundary value dynamic model, Iteration, Optimal control, Sensitivity analysis.

\begin{abstract}
In this paper, the mathematical modeling of the country race in 2014 title A - Chang E III aircraft lunar soft landing problem for background, determined the location of nearly Apo lune, and solved various stages of parameters to obtain a series of reasonable conclusions. First, the use of two-point boundary value dynamics model, discrete continuous process, solved for the location of nearly Apolune. Then analyzing the various stages of the soft landing, the key point is to state constraints to optimize the index to establish minimum energy optimal control model, and iterative calculations to get the aircraft away from the lunar surface height and quality changes over time case. The minimum energy consumption was 1.16t. And finally, do a sensitivity analysis to verify that the system has strong stability and robustness.
\end{abstract}

\section{Introduction}

The soft landing problem of Chang'e 3 spacecraft is a very complex, particularly changes in specific parameters of the middle each phase is more complicated, and this paper mainly adopts a simple iterative thinking to achieve physical implementation of various complex phases, the most optimal control strategy has obtained.

\section{The position determination of perilune and apolune}

The scheduled landing point position Chang'e 3 has been known, by using the inverse method, by universal gravitation formula, we can get perilune speed[1], the dynamic model of two-point boundary value is adopted to solve the position of perilune and apolune, because the horizontal migration resulted from late obstacle avoidance is relatively small, ignoring the impact, only considering the migration of main retard phase, the following dynamic model of two-point boundary value is obtained:

$$
\left\{\begin{array}{l}
a_{x}=\frac{-F \cos (\partial)}{m} \\
a_{y}=1.63 a-\frac{F \sin (\partial)}{m} \\
\mathrm{~s}=\mathrm{s}_{s}+v_{x} \Delta t+\frac{1}{2} a_{x} \Delta t^{2} \\
h=h_{s}+v_{y} \Delta t+\frac{1}{2} a_{y} \Delta t^{2}
\end{array}\right.
$$

Among them, $\partial$ is thrust Angle, $h$ is dropping height, $s$ is the horizontal movement distance, $m$ is the quality of Chang'e 3. And in perilune should meet the following relations: $v_{x}=1672.359$, $m=2400$, the rest $v_{y}, \partial, s, h$ are 0 .

The main retard phase time is $T$, in the case of ensuring and meeting the constraint conditions, it will adopts thrust equivalent to constant force [3], throughout the process, $F, T$ are unknown quantity, which meet the following relations:

$0<T<750$

$1500 \leq F \leq 7500$ 
Because the thrust in the main retard phase is time-varying, and $m$ is also a real-time change, so we will divide the whole process into multiple small time period $\Delta t$ to research, that is numerical method, by adopting iterative idea, by programming to work out Time-consuming of the main retard phase $T=$ 515 seconds, equivalent thrust is $5820 \mathrm{~N}$, dropping height in vertical direction is $12.02 \mathrm{~km}$, fuel consumption in main retard phase is $1.02 \mathrm{t}$, after main retard phase the quality Chang 'e 3 is $1.38 \mathrm{t}$, horizontal movement distance of Chang'e in main retard phase is $481.33 \mathrm{~km}$, and the position of perilune and apolune are available.

\section{The optimization and optimal control of soft-landing flight orbit}

The above mainly analyzes the change condition of specific parameters in the main retard phase, and now discuss the following five phases, and then get the best control strategy.

(1) Rapid adjustment phase, the thrust and direction are undefined, so random simulation method is used to solve energy consumption optimization problem in this phase. $F$ adopts a random value between $1500 \mathrm{~N}-7500 \mathrm{~N}$, updated thrust value of $\mathrm{F}$ every 0.5 seconds, and component force in the horizontal direction adopts $F \times$ rand , the component force in the vertical direction is determined by the Pythagorean theorem, then iterating according to constraint condition in the model, until the difference between termination condition meet within the error value, the program can be run repeatedly to obtain optimal solution.

(2) The parabolic movement phase of rough obstacle avoidance phase, we can adopt the similar method in rapid adjustment phase, the optimal results are obtained; In vertical dropping phase, the terminal time is distant from the lunar surface is $100 \mathrm{~m}$ as constraint condition, $v_{y}$ is infinitely close to 0 as out of the loop condition and critical conditions, the force of satellite in this phase is constrained to the iterative procedure, finally the equivalent constant value $F=2778.5$ is available in this phase. Rough obstacle avoidance vertical dropping phase takes 77.5 seconds.

(3) Hovering phase, after checking the relevant information of Chang'e 3, this phase lasts for 30 seconds, after hovering the quality of Chang'e 3 is $1.25 \mathrm{t}$.

(4) Detailed obstacle avoidance phase (excluding other parts of the hovering phase) and slow dropping stage, the results can be obtained by using the simplified model.

(5) Free falling phase, the thrust engine does not work, the engine without energy consumption, the quality of Chang'e 3 remains the same.

Table 1 Parameter values after the completion of each phase

\begin{tabular}{|c|c|c|c|c|}
\hline Phase type & Time (second) & $\begin{array}{c}\text { The distance to } \\
\text { the lunar surface } \\
\text { (meter) }\end{array}$ & Quality (ton) & $\begin{array}{c}\text { Vertical speed } \\
\text { (meter per } \\
\text { second) }\end{array}$ \\
\hline $\begin{array}{c}\text { Rapid } \\
\text { adjustment phase }\end{array}$ & 11.5 & 2398.4 & 1.3702 & 47.2 \\
\hline $\begin{array}{c}\text { Rough obstacle } \\
\text { avoidance phase }\end{array}$ & 95 & 99.93 & 1.27 & 0.06 \\
\hline $\begin{array}{c}\text { Hovering phase } \\
\text { Detailed obstacle } \\
\text { avoidance phase }\end{array}$ & 30 & 99.93 & 1.25 & 0 \\
\hline $\begin{array}{c}\text { Free falling } \\
\text { phase }\end{array}$ & 23 & 3.96 & 1.24 & 0 \\
\hline
\end{tabular}


According to the above results, the model are done analogue, the distance from Chang'e 3 to lunar surface changes over time as below figure:

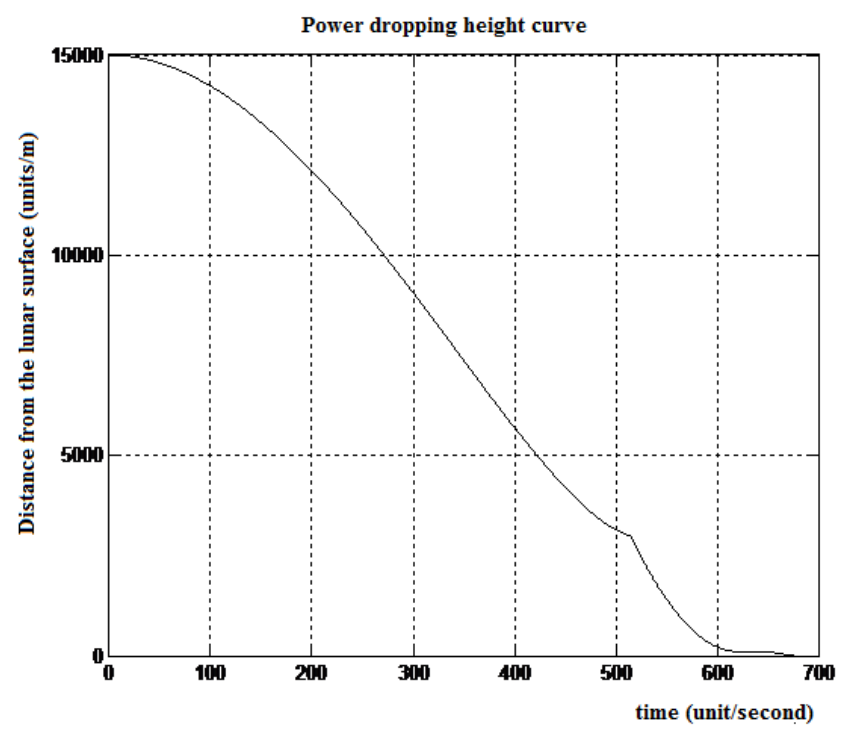

Fig.1 The height from lunar surface change over time

It is known from Fig.1, the power dropping phase of Chang'e 3 took 676.5 seconds, after landing, the quality is $1.237 \mathrm{t}$, the total energy consumption from perilune to landing is $1.163 \mathrm{t}$, which is the minimum energy consumption, energy consumption is $48.46 \%$ of the initial mass.

\section{Sensitivity analysis of model}

In question 1 , we choose $F=5820 \mathrm{~N}$ as constant thrust of our equivalent effect, now extend the limitation condition of the first question, get a near value around $F$, take $F=5770$ N at this time, $F$ is done the same change process, also get a $F, t$ trajectory, compared with the original trajectory as follows:

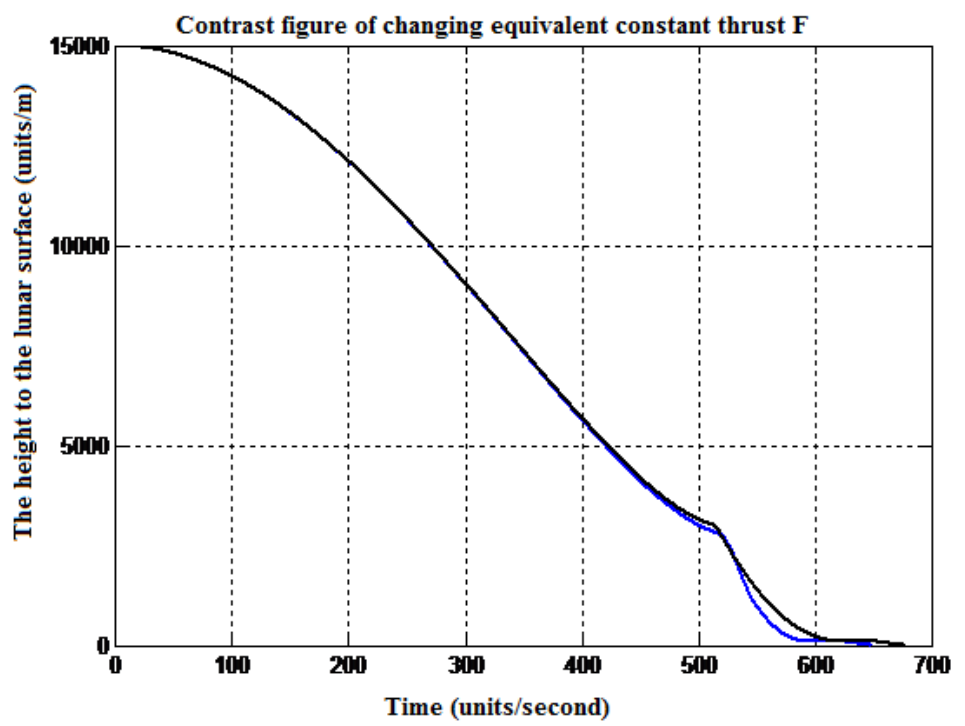

Fig.2 The height contrast figure of changing constant thrust

It is known from Fig.2, high trajectory basically coincide, it shows thrust has changed, height change of Chang'e 3 is not sensitive to the selection of thrust, so the system has very strong robustness. 


\section{Conclusion}

This paper studies specific process various phase of the Chang'e 3 spacecraft in soft landing, with relatively simple iterative idea, realize the optimal control strategy of Chang'e 3 soft landing, finally the sensitivity analysis has carried on, verified the accuracy of results, which has the certain academic reference value.

\section{References}

[1] Li Xing. Perihelion and aphelion speed of two kinds of typical solutions [J].Science problem solving research, 2013, (6): 48.

[2] Wang Datie, Li Tieshou, appl. Math. Mech. Vol.11. The numerical method for solving the optimal soft landing of two-point boundary value problems [J]. Journal of control engineering, 2000 (3): 7-11.

[3] Wang Jie, Cui Naigang, liu Tun, mr.li. Constant thrust lunar lander optimal soft landing trajectory study [J]. High technology communication, 2003, 13(4): 39-42.

[4] Jiang Rui, Han Bing. Chang'e 3 landing control research and software simulation[J]. Microcomputer applications, 2012, 28(2): 17-34. 